INPLASY

PROTOCOL

To cite: Llorente-Pérez et al. Educational Interventions for the Prevention and Control of Dengue in Adults: An Integrative Review Protocol. Inplasy protocol 202170038. doi:

10.37766/inplasy2021.7.0038

Received: 12 July 2021

Published: 12 July 2021

Corresponding author:

Yolima Judith Llorente Pérez

yolima.llorente@udea.edu.co

Author Affiliation:

Universidad de Antioquia.

Support: None.

Review Stage at time of this submission: Preliminary

searches.

Conflicts of interest:

None declared.

\section{Educational Interventions for the Prevention and Control of Dengue in Adults: An Integrative Review Protocol}

Llorente-Pérez, YJ1; Rodríguez-Acelas, AL2; Cañon-Montañez, W33.

Review question / Objective: To review and synthesize the evidence of educational interventions for the prevention and control of dengue in adults.

Condition being studied: Dengue is a disease of public health interest, it generates great concern worldwide due to the high burden it represents, generating disabilities, death, social and economic effects. The increase in cases can be attributed to social, demographic, environmental changes or factors facilitated by the community. To deal with dengue, different strategies have been established from health education, educational interventions being necessary tools to reduce the disease. The development of educational interventions seeks to generate commitments on the part of the adult population towards the prevention and control of the disease.

INPLASY registration number: This protocol was registered with the International Platform of Registered Systematic Review and Meta-Analysis Protocols (INPLASY) on 12 July 2021 and was last updated on 12 July 2021 (registration number INPLASY202170038).

\section{INTRODUCTION}

Review question / Objective: To review and synthesize the evidence of educational interventions for the prevention and control of dengue in adults.

Condition being studied: Dengue is a disease of public health interest, it generates great concern worldwide due to the high burden it represents, generating disabilities, death, social and economic effects. The increase in cases can be attributed to social, demographic, environmental changes or factors facilitated by the community. To deal with dengue, different strategies have been established from health education, 
educational interventions being necessary tools to reduce the disease. The development of educational interventions seeks to generate commitments on the part of the adult population towards the prevention and control of the disease.

\section{METHODS}

Search strategy: The search will be carried out in the following databases: MEDLINE (via PUBMED), LILACS, Web of Science and EMBASE. The following descriptors, terms and keywords will be combined for the search strategy: Adult, Aged, Middle Aged, Health Education, Community Health Education, Control Group, Standard Care, Usual Care, Dengue, Dengue Virus, Prevention, Control.

Participant or population: Adults of both sexes.

Intervention: Educational interventions.

Comparator: Usual care, standard care or control group.

Study designs to be included: Intervention studies.

Eligibility criteria: Randomized controlled trials (RCTs), quasi-experimental or nonrandomized studies and cluster studies, published in any language, from inception until March 31, 2021, with abstract and full text available, will be included. Editorial letters, conference abstracts and duplicate studies will be excluded.

Information sources: Electronic databases: EMBASE, MEDLINE (via PUBMED), Web of Science and LILACS.

Main outcome(s): Dengue prevention and control.

Data management: The following variables will be extracted from the studies: author, year, country, study design, sample size, intervention group, control group, type of intervention, and main findings.
Quality assessment / Risk of bias analysis: The methodological quality for randomized clinical trials and cluster studies will be assessed using the Cochrane Collaboration tool (RoB 1.0) to assess risk of bias in randomized trials. The Cochrane Collaboration risk of bias tool for RCTs is available in RevMan 5.4. For quasiexperimental studies, the Study Quality Assessment Tool from National Heart, Lung, and Blood Institute, will be used. The level of evidence in the studies will be evaluated, according to the considerations or guidelines of The Joanna Briggs Institute (JBI).

Strategy of data synthesis: A narrative synthesis of the findings of the studies of educational interventions for the prevention and control of dengue in adults will be carried out.

Subgroup analysis: Not applicable.

Sensitivity analysis: Not applicable.

Country(ies) involved: Colombia.

Keywords: Dengue, Health Education, Prevention and Control.

Dissemination plans: The journal for the publication of the review manuscript has not yet been determined.

Contributions of each author:

Author 1 - Yolima Judith Llorente Pérez.

Email: yolima.llorente@udea.edu.co

Author 2 - Alba Luz Rodríguez-Acelas.

Email: aluz.rodriguez@udea.edu.co

Author 3 - Wilson Cañon-Montañez.

Email: wilson.canon@udea.edu.co 